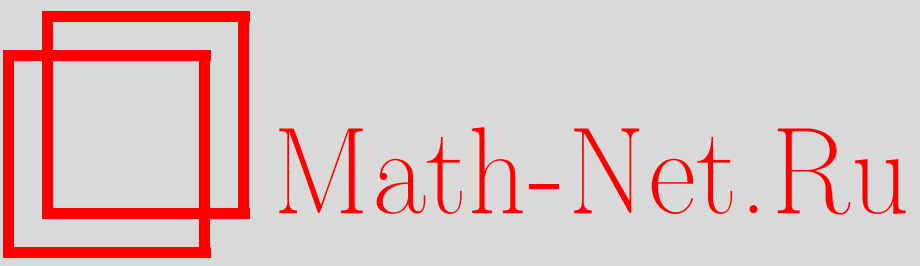

О. И. Мохов, Интегрируемые бигамильтоновы системы гидродинамического типа, УМH, 2002, том 57, выпуск 1, 157-158

DOI: https://doi.org/10.4213/rm483

Использование Общероссийского математического портала Math-Net.Ru подразумевает, что вы прочитали и согласны с пользовательским соглашением

http://www.mathnet.ru/rus/agreement

Параметры загрузки:

IP: 3.89.197.203

26 апреля 2023 г., 03:35:59 


\title{
ИНТЕГРИРУЕМЫЕ БИГАМИЛЬТОНОВЫ СИСТЕМЫ ГИДРОДИНАМИЧЕСКОГО ТИПА
}

\author{
О.И. Мохов
}

В данной заметке построена иерархия интегрируемых бигамильтоновых систем, обладающих парой согласованных локальных скобок Пуассона гидродинамического типа (согласованных скобок Дубровина-Новикова [1]) и задаваемых произвольными решениями нелинейной системы уравнений, интегрируемой методом обратной задачи рассеяния и описьвающей все согласованные локальные скобки Пуассона гидродинамического типа.

Напомним, что локальной заменой координат произвольная пара согласованных гамильтоновых операторов Дубровина-Новикова $P_{1}^{i j}$ и $P_{2}^{i j}$, один из которых (допустим $P_{2}^{i j}$ ) невырожденный, приводится к следующему специальному виду:

$$
\begin{aligned}
& P_{2}^{i j}[v(x)]=\eta^{i j} \frac{d}{d x}, \\
& P_{1}^{i j}[v(x)]=\left(\eta^{i s} \frac{\partial h^{j}}{\partial v^{s}}+\eta^{j s} \frac{\partial h^{i}}{\partial v^{s}}\right) \frac{d}{d x}+\eta^{i s} \frac{\partial^{2} h^{j}}{\partial v^{s} \partial v^{k}} v_{x}^{k},
\end{aligned}
$$

где $\left(\eta^{i j}\right)$ - произвольная невырожденная постоянная симметричная матрица: $\operatorname{det}\left(\eta^{i j}\right) \neq 0, \eta^{i j}=$ const, $\eta^{i j}=\eta^{j i} ; h^{i}(v), 1 \leqslant i \leqslant N$, - гладкие функции, заданные в некоторой области локальных координат, такие, что оператор (2) является гамильтоновым (см. [2], [3]). При этом оператор вида $(2)$ является гамилтоновым тогда и только тогда, когда

$$
\begin{gathered}
\eta^{s r} \frac{\partial^{2} h^{j}}{\partial v^{s} \partial v^{i}} \frac{\partial^{2} h^{k}}{\partial v^{l} \partial v^{r}}=\eta^{s r} \frac{\partial^{2} h^{k}}{\partial v^{s} \partial v^{i}} \frac{\partial^{2} h^{j}}{\partial v^{l} \partial v^{r}} \\
\left(\eta^{i p} \frac{\partial h^{s}}{\partial v^{p}}+\eta^{s p} \frac{\partial h^{i}}{\partial v^{p}}\right) \eta^{j l} \frac{\partial^{2} h^{r}}{\partial v^{l} \partial v^{s}}=\left(\eta^{j p} \frac{\partial h^{s}}{\partial v^{p}}+\eta^{s p} \frac{\partial h^{j}}{\partial v^{p}}\right) \eta^{i l} \frac{\partial^{2} h^{r}}{\partial v^{l} \partial v^{s}}
\end{gathered}
$$

(см. [2]-[5]). Система нелинейных уравнений (3), (4), как и предполагалось в работах автора [4], [5], где были высказаны соответствующие гипотезы, является интегрируемой методом обратной задачи рассеяния. Процедура интегрирования этой системы была предложена в работе автора [6], [7], а в работе [8] была указана для нее пара Лакса. Отметим, что уравнения ассоциативности двумерной топологической теории поля (см. [2]) являются естественной редукцией уравнений (3) для "потенциальных" векторных полей $h^{i}(v), 1 \leqslant i \leqslant N$, специального вида $h^{i}(v)=\eta^{i j} \partial \Phi / \partial v^{j}$, где $\Phi(v)$ - некоторая гладкая функция ("потенциал") (см. также [3]-[5], [9]).

Рассмотрим оператор рекурсии, задаваемшій "каноническими" согласованными гамильтоновьми операторами Дубровина-Новикова (1), (2):

$$
R_{l}^{i}=\left[P_{1}[v(x)]\left(P_{2}[v(x)]\right)^{-1}\right]_{l}^{i}=\left(\left(\eta^{i s} \frac{\partial h^{j}}{\partial v^{s}}+\eta^{j s} \frac{\partial h^{i}}{\partial v^{s}}\right) \frac{d}{d x}+\eta^{i s} \frac{\partial^{2} h^{j}}{\partial v^{s} \partial v^{k}} v_{x}^{k}\right) \eta_{j l}\left(\frac{d}{d x}\right)^{-1}
$$

где $\left(\eta_{i j}\right)$ - матрица, обратная по отношению к матрице $\left(\eta^{i j}\right): \eta^{i s} \eta_{s j}=\delta_{j}^{i}$. Применим полученный нами оператор рекурсии (5) к системе трансляций по $x$, т.е. системе гидродинамического типа $v_{t}^{i}=v_{x}^{i}$, которая, очевидно, является гамильтоновой с гамильтоновым оператором (1):

$$
v_{t}^{i}=v_{x}^{i} \equiv P_{2}^{i j} \frac{\delta H}{\delta v^{j}(x)}, \quad H=\frac{1}{2} \int \eta_{j l} v^{j}(x) v^{l}(x) d x .
$$

Работа вьполнена при финансовой поддержке Фонда Александра фон Гумбольта (Германия), а также Российского фонда фундаментальных исследований (грант № 99-01-00010) и фонда INTAS (грант № 99-1782). 
Любая система из иерархии

$$
v_{t_{n}}^{i}=\left(R^{n}\right)_{j}^{i} v_{x}^{j}, \quad n \in \mathbb{Z},
$$

является мультигамильтоновой интегрируемой системой.

В частности, интегрируемой является любая система вида $v_{t_{1}}^{i}=R_{j}^{i} v_{x}^{j}$, т.е. система гидродинамического типа

$$
\begin{aligned}
v_{t_{1}}^{i} & =\left(\left(\eta^{i s} \frac{\partial h^{j}}{\partial v^{s}}+\eta^{j s} \frac{\partial h^{i}}{\partial v^{s}}\right) \frac{d}{d x}+\eta^{i s} \frac{\partial^{2} h^{j}}{\partial v^{s} \partial v^{k}} v_{x}^{k}\right) \eta_{j l} v^{l} \\
& \equiv\left(\eta^{i s} \frac{\partial h^{j}}{\partial v^{s}} \eta_{j k}+\frac{\partial h^{i}}{\partial v^{k}}+\eta^{i s} \eta_{j l} \frac{\partial^{2} h^{j}}{\partial v^{s} \partial v^{k}} v^{l}\right) v_{x}^{k} \equiv\left(h^{i}(v)+\eta^{i s} \frac{\partial h^{j}}{\partial v^{s}} \eta_{j l} v^{l}\right)_{x}
\end{aligned}
$$

где $h^{i}(v), 1 \leqslant i \leqslant N,-$ произвольные решения интегрируемой системы (3), (4).

Эта система гидродинамического типа бигамильтонова с парой "канонических" гамильтоновых операторов Дубровина-Новикова (1), (2):

$$
\begin{gathered}
v_{t_{1}}^{i}=\left(\left(\eta^{i s} \frac{\partial h^{j}}{\partial v^{s}}+\eta^{j s} \frac{\partial h^{i}}{\partial v^{s}}\right) \frac{d}{d x}+\eta^{i s} \frac{\partial^{2} h^{j}}{\partial v^{s} \partial v^{k}} v_{x}^{k}\right) \frac{\delta H_{1}}{\delta v^{j}(x)}, \quad H_{1}=\frac{1}{2} \int \eta_{j l} v^{j}(x) v^{l}(x) d x \\
v_{t_{1}}^{i}=\eta^{i j} \frac{d}{d x} \frac{\delta H_{2}}{\delta v^{j}(x)}, \quad H_{2}=\int \eta_{j k} h^{k}(v(x)) v^{j}(x) d x
\end{gathered}
$$

Следующей системой в иерархии (7) является интегрируемая система гидродинамического типа

$$
\begin{aligned}
v_{t_{2}}^{i}= & \left(\left(\eta^{i s} \frac{\partial h^{j}}{\partial v^{s}}+\eta^{j s} \frac{\partial h^{i}}{\partial v^{s}}\right) \frac{d}{d x}+\eta^{i s} \frac{\partial^{2} h^{j}}{\partial v^{s} \partial v^{k}} v_{x}^{k}\right) \eta_{j l}\left(h^{l}(v)+\eta^{l p} \frac{\partial h^{r}}{\partial v^{p}} \eta_{r q} v^{q}\right) \\
\equiv & \left(\left(\eta^{i s} \frac{\partial h^{j}}{\partial v^{s}}+\eta^{j s} \frac{\partial h^{i}}{\partial v^{s}}\right)\left(\eta_{j l} \frac{\partial h^{l}}{\partial v^{k}}+\eta_{r k} \frac{\partial h^{r}}{\partial v^{j}}+\eta_{r q} v^{q} \frac{\partial^{2} h^{r}}{\partial v^{j} \partial v^{k}}\right)\right. \\
& \left.+\eta^{i s} \frac{\partial^{2} h^{j}}{\partial v^{s} \partial v^{k}}\left(\eta_{j l} h^{l}(v)+\eta_{r q} v^{q} \frac{\partial h^{r}}{\partial v^{j}}\right)\right) v_{x}^{k}
\end{aligned}
$$

Иерархия интегрируемых систем (7) является “канонической” для всех бигамильтоновых систем гидродинамического типа, обладающих парой согласованных локалшных гамилтоновых операторов гидродинамического типа. Полностью аналогичная явная конструкция класса интегрируемых бигамильтоновых систем построена нами и в случае нелокальных согласованных гамильтоновых операторов гидродинамического типа.

\section{СПИСОК ЛИТЕРАТУРЫ}

[1] Б. А. Дубровин, С. П. Новиков // Докл. АН СССР. 1983. Т. 270. № 4. С. 781-785. [2] B. Dubrovin // Lecture Notes in Math. 1996. V. 1620. P. 120-348; arXiv: hep-th/9407018. [3] О. И. Мохов // УМН. 1997. Т. 52. № 6. С. 171-172. [4] О. И. Мохов // Тр. МИРАН. 1999. T. 225. C. 284-300. [5] O. I. Mokhov // Reports Math. Physics. 1999. V. 43. № 1/2. P. 247-256. [6] O. I. Mokhov. arXiv: math.DG/0005081. [7] О. И. Мохов // УМН. 2001. Т. 56. № 2. C. 221-222. [8] E. V. Ferapontov. arXiv: math.DG/0005221. [9] О. И. Мохов // УМН. 1998. T. 53. № 2. C. 153-154.

Центр нелинейных исследований при Институте теоретической физики им. Л. Д. Ландау РАН 